\title{
NONLINEAR WAVES IN FLUX TUBES
}

\author{
MRYUTOVA \\ 630090 \\ Novosibirsk, 90 \\ USSR
}

\begin{abstract}
Some results of nonlinear studies of flux tube oscillations are summarized showing that each step made towards the real conditions of the solar atmosphere opens the existence of qualitatively new effects which form the basis of strongly nonlinear dynamics. Among these effects are, for example: explosive instability in the presence of negative energy waves which leads to an efficient and fast energy release; the generation of secondary plasma flows and current drive which strongly influence the evolution of magnetic flux tubes and their stability conditions; formation of shocks and solitary waves, temporal brightening of flux tubes and others.
\end{abstract}

\section{Introduction}

Here, in Bangalore, we could celebrate the 30th anniversary of the suggestion by Robert Howard (1959) that the solar magnetic field can be concentrated in bundles of field lines. After the first direct observations by Sheeley (1967) there has been no lack of new observational data; there were results of Beckers and Schröter (1968), Tanenbaum et al. (1969), Livingston and Harvey (1969), Frazier (1971), Grigorjev and Kuklin (1971), Gopasyuk and Tsap (1971), Howard and Stenflo (1972) and others (for more details see, for example, Stenflo (1989)). Even this very incomplete list of explorers shows the exciting and great activity of the observers, which was the beginning of a new period in Solar Physics - the physics of structured magnetic fields. For understanding the basic processes in the solar atmosphere, which are obviously governed by magnetic fields, the properties of separate magnetic flux tubes as well as the properties of their ensembles should be analysed. Beside its significance for different astrophysical objects where structured magnetic fields are met quite often, such a study is also of interest as a fundamental problem of plasma physics due to a rich picture of wave processes and instabilities in such structures. For the time being we have a good theoretical experience as well due to the essential contribution by $\mathrm{P} R$ Wilson, L E Cram, E Parker, W Unno, N Weiss, S Syrovatskii, J Uberoi, E R Priest and others (see Priest, 1982 and References therein).

Today there is a rather good understanding of the properties of linear oscillations of a single flux tube: eigenmodes, mechanisms of damping, effects of vertical stratification and so on. Even in the simplest model of a homogeneous tube the physics of flux tubes is very rich. Motions in the convective zone and, as will be shown below, strong shear flows excite different kinds of oscillations of a flux tube. Among these the most important are longwave oscillations (with $k R \ll<$, where $k=1 / \lambda$ is the wave number and $R$ is the tube radius) corresponding to a dipole mode (with an azimuthal wave number $m= \pm 1$ ) that has bending or kink oscillations and an axisymmetric sausage mode $(m=0)$. For both modes the frequency scales linearly with wave number.

The bending mode is an analogue of the Alfven wave with the phase velocity containing the density of the ambient plasma which is caused by the effect of «added mass» (Ryutov and Ryutova, 1976; Spruit, 1981):

$$
c_{b}=\frac{\omega}{k_{z}}=\frac{a}{\sqrt{1+1 / \eta}},
$$

where $\eta=\rho_{i} / \rho_{e}, \rho$ is the plasma density, $a=b / 4 \pi \rho_{i}$ is the Alfven velocity. Here and below the subscripts $i$ and e correspond to the regions inside and outside the flux tube. The phase velocity (1) is written assuming that there is no magnetic field outside the tube. 
The sausage mode is the analogue of the slow magnetosonic wave. In these oscillations the sum of gas-kinetic and magnetic pressures is almost not perturbed, so that the plasma parameters outside the tube have little influence on their dispersion relation (Defouw, 1976):

$$
c_{T}=\frac{\omega}{k_{z}}=\frac{a s_{i}}{\sqrt{a^{2}+s_{i}^{2}}}
$$

Here $s^{2}=\gamma p / p$ is a sound speed squared ( $\gamma$ is a specific heat ratio). As to the oscillations with higher azimuthal mode numbers $m= \pm 2, \pm 3, \ldots$ they are weakly coupled with the larger-scale motions of the atmosphere (their amplitudes are proportional to $(k R)^{m}$ ) and are of relatively less interest.

Of course, in a magnetic flux tube as in any oscillating system there is present a standard set of nonlinear phenomena like shocks, solitons, collapse etc. Besides that, there are the specific effects caused by the peculiarity of the flux tube itself. Before proceeding to some results of nonlinear studies of flux tube oscillations we will describe in the next Section some features of flux tubes which are important for understanding more complicated nonlinear phenomena.

\section{Nondissipative Damping Mechanisms}

In a compressible medium an oscillating magnetic flux tube can be a source of secondary acoustic waves (Ryutov and Ryutova, 1976). In general, if outside the tube there is an external magnetic field the tube radiates all types of MHD-waves. The radiation takes place in the case when the phase velocity of oscillations is larger than the sound speed in the ambient plasma, that is when the radial wave number

$$
k_{r}=\frac{1}{s_{e}}\left(\omega^{2}-k^{2} s_{e}^{2}\right)^{1 / 2}
$$

is real. In the opposite case the radiated waves are evanescent and their presence leads only to a small change of phase velocity. The radiative damping rate caused by this effect for an arbitrary mode (except $m=0$ ) is as follows:

$$
\alpha_{\mathrm{rd}}^{(\mathrm{m})}=\frac{\pi \omega}{|\mathrm{m}| !(|\mathrm{m}|-1) !(1+\eta)} \frac{(\mathrm{kR})^{2|\mathrm{~m}|}}{2}\left[\frac{2}{\gamma(1+\eta)-1}\right]^{\mid \mathrm{ml}} .
$$

Respectively, for the kink mode we have

$$
\alpha_{\mathrm{rad}}^{(1)}=\frac{\pi \omega}{1+\eta} \frac{(\mathrm{kR})^{2}}{2}\left[\frac{2}{\gamma(1+\eta)}-1\right] .
$$

Note, that the natural frequencies of tube oscillations are independent of azimuthal mode number and are of the same form as expression (1) for all $|\mathrm{m}|>1$.

The radiative damping works in the case of sausage oscillations as well. The corresponding damping rate is (Ryutova, 1981)

$$
\alpha_{\mathrm{rad}}^{(0)}=\frac{\pi}{2}\left(\frac{k R}{2}\right)^{2} \frac{\omega s_{i}^{6}}{s_{e}^{2}\left(a^{2}+s_{i}^{2}\right)^{2}}
$$

The damping rate contains for all cases the small parameter $(\mathrm{kR})^{2}$ and is typically small, which means that once excited these oscillations propagate along the flux tube. The 
situation becomes quite different in the more realistic case of smooth radial profiles of density and magnetic field inside the tube.

In the case of a radially inhomogeneous tube there exists another nondissipative mechanism of damping connected with the pumping of the oscillation energy into the resonance layer where the phase velocity of oscillations becomes equal to the local Alfven velocity. The mathematical form of the corresponding equation is of the Rayleigh equation in usual hydrodynamics with the coefficient of the higher derivative approaching zero at the singular point. The general features of such a type of equation and, in particular, Alfven resonances in laboratory plasmas were studied by many authors (Timofeev, 1970; Tataronis and Grossman, 1973; Chen and Hasegawa, 1974; Timofeev, 1989). It was explored as well in connection with the physics of coronal loops and heating processes in the solar atmosphere (Ionson, 1978; Hollweg, 1979; Heyvaerts and Priest, 1983; Sudan and Similon, 1988).

The resonance damping of bending oscillations of a magnetic flux tube was first studied in a model of a tube with diffuse boundaries (Ryutova, 1977). It was assumed that the magnetic field is almost constant across the whole tube except in a narrow region of width $l<<\mathrm{R}$ where it linearly decreases to zero. Respectively, the dependence of pressure and plasma density follows the magnetic field in accordance with the equilibrium condition. In this approach the resonance damping rate of bending oscillations is as follows:

$$
v_{\text {res }}=\frac{\pi \omega}{4} \frac{\rho_{i}}{\rho_{i}+\rho_{e}} \frac{l}{R}
$$

For the slow (sausage) oscillations resonance absorption does not take place, but the radial inhomogeneity plays a crucial role: these oscillations appear to be very sensitive to inhomogeneities of plasma and magnetic field across the tube and experience a strong radiative damping (Ryutova, 1981). To be more precise, if the relative drop of plasma density and/or magnetic field over the tube radius exceeds the magnitude (kR) ${ }^{2}$ (which is extremely small for longwave oscillations) the sausage oscillations vanish. In the case of a really smooth radial dependence of flux tube parameters the weakly damped wave with smoothly varying radial eigenfunction does not exist any more. And the question arises of whether under such conditions the oscillations of flux tubes can still be the agent responsible for energy transfer from the lower to upper layers of the atmosphere. The answer is positive (Ryutova and Khijakadze, 1989). This is connected with the fact that the resonance damping in a smoothly inhomogeneous tube gives rise to a pecular evolution of the radial mode structure of the perturbation: it becomes more and more spiky at higher altitudes. The characteristic radial scale length $\Delta r$ diminishes inversely proportional to $z$ :

$$
\Delta \mathrm{r} \sim \mathrm{aR} / \omega z .
$$

Respectively, due to the presence of the small-scale structure the usual dissipative processes (viscosity, thermal conductivity, ets.) become important at larger $z$. The longitudinal dependence of the energy flux of oscillations becomes nonexponential. For example, the volume density of power $Q$ released by viscous dissipation is of the order of

$$
Q \sim \rho v \frac{\omega^{2} \xi^{2}}{(\Delta r)^{2}}
$$

( $v$ is a kinematic viscosity coefficient and $\xi$ is the tube's displacement). As $\Delta r$ diminishes with height $Q$ is growing with $z$. At some altitude $z^{\circ}, Q$ reaches a maximum, and then rapidly decreases. Thus, the heating power has a pronounced maximum at some altitude $\mathbf{z}^{*}$, which can estimated as

$$
z^{*} \sim \frac{\mathrm{a}(\mathrm{r})}{\omega}\left(\frac{\omega \mathrm{R}^{2}}{v}\right)^{1 / 3} .
$$


In the observational data this effect can manifest itself by a temporal brightening of the flux tube region at a definite height.

\section{Effects of Sheared Flows}

According to the observational data there are plasma flows along magnetic structures with different velocities inside and outside them.

The presence of shear flows along the magnetic fields leads to the appearance of qualitatively new effects. First of all, to the existence of negative energy waves, and thus to a widening of the classes of instability. And second, to a linear hydrodynamic instability which is similar to a tangential discontinuity and which can be responsible for the excitation of flux tube oscillations in regions far from the convective zone.

The specific features of negative energy waves are that the energy of a system with these waves is lower than without them and losing the energy of negative energy waves due to dissipative effects (say, the interaction with the waves of positive energy, with particles and so on) leads to a growth of their amplitudes (Kadomtsev et al., 1964; Dikasov et al., 1965; Coppi et al., 1969; Weiland and Wilhelmson, 1977).

In a cylindrical coordinate system with the $\mathrm{z}$-axis directed along the flux tube the linearized MHD-equations in the presence of shear flow with a velocity $u(r)$ are as follows (Ryutova, 1988):

$$
\begin{aligned}
& i \delta \mathcal{P}=\rho(r) \frac{\Omega^{2}(r)\left(s^{2}+a^{2}\right)-k^{2} s^{2} a^{2}}{\Omega^{2}-k^{2} s^{2}}\left[\frac{1}{r} \frac{\partial}{\partial r} r \frac{v_{r}}{\Omega(r)}+\frac{i m}{r} \frac{v_{\varphi}}{\Omega(r)}\right], \\
& \frac{\partial \delta \mathcal{P}}{\partial r}=i \rho(r)\left[\Omega^{2}(r)-k^{2} a^{2}\right] \frac{v_{r}}{\Omega(r)}, \\
& \frac{i m_{r}}{r} \mathcal{P}=i \rho(r)\left[\Omega^{2}(r)-k^{2} a^{2}\right] \frac{v_{r}}{\Omega(r)} .
\end{aligned}
$$

Here $\delta \mathcal{P}=\delta p+b_{z} B / 4 \pi$ is the total pressure perturbation (all perturbed quantities are assumed to be proportional to $\exp (-\mathrm{i} \omega t+\mathrm{ikz}+\mathrm{im} \varphi))$, and

$$
\Omega(r)=\omega-k u(r) .
$$

The set (6) describes all the types of linear oscillations of magnetic flux tubes in the presence of arbitrary shear flows along the magnetic field.

As an example we present here the results for a kink mode in the simplest model of shear flow without a radial profile (taking into account a radial profile of flows near the tube boundary leads to very interesting physics and will be presented elsewhere). In this case the set $(6)$ is reduced to the following equation for the tube's displacement vector $\vec{\xi}$ :

$$
\rho_{i} \frac{\partial^{2} \vec{\xi}}{\partial t^{2}}=-\rho_{e}\left(\frac{\partial}{\partial t}+\frac{\partial}{\partial z}\right)^{2} \vec{\xi}+\frac{B^{2}}{4 \pi} \frac{\partial^{2} \vec{\xi}}{\partial z^{2}} .
$$

The dispersion relation corresponding to (7) has a form:

$$
\omega^{2}+\frac{1}{\eta}(\omega-k u)^{2}-k^{2} a^{2}=0
$$

From (8) we have 


$$
\frac{\omega}{k}=\frac{1}{1+\eta}\left\{u \pm \sqrt{\eta\left[a^{2}(1+\eta)-u^{2}\right]}\right\} .
$$

One can see from (9) that if the velocity of the shear flow exceeds the threshold

$$
u>a \sqrt{1+\eta}
$$

the system becomes unstable: this is a linear hydrodynamic instability similar to the instability of a tangential discontinuity. This instability may play an essential role in the excitation of oscillations in magnetic structures in the chromosphere and corona: if somewhere the magnetic flux tube is «blown oven» by the upstream flow, the oscillations excited there will subsequently propagate upstream. The frequency of these oscillations is of course unrelated to time scales in the convection zone and may be much greater than the reciprocal of the time for granulation.

In the region $u<a \sqrt{1+\eta}$ where the system is stable with respect to TD-instability the instability of negative energy waves can still develop. The direct calculations for the energy density of waves corresponding to the lower branch («minus» sign) of the dispersion relation (9) give:

$$
W=\pi R^{2} k^{2} \rho_{e} \xi^{2}\left(\eta a^{2}-u^{2}\right) \frac{x}{x+u},
$$

where $x=\sqrt{\eta\left[a^{2}(1+\eta)-u^{2}\right]}$. So, at flow velocities exceeding the threshold

$$
u>a \sqrt{\eta}
$$

the system becomes unstable with respect to negative energy waves. The interval $a \sqrt{\eta}<u<a \sqrt{1+\eta}$ where the instability of negative energy waves can exist can be written in terms of $\beta=8 \pi \mathrm{p} / \mathrm{B}^{2}$ :

$$
a \sqrt{\frac{\beta}{1+\beta}}<u<a \sqrt{\frac{1+2 \beta}{1+\beta}} .
$$

For the development of the instability the dissipative processes must turn on. In other words, incorporating dissipative effects results in a transfer of energy away from the negative-energy waves and an increase in their amplitude. A remarkable property which a magnetic tube exhibits is that even in the absence of the usual dissipative processes this instability can develop due to the collisionless dissipation mechanisms described in Sec. 2, namely, due to the radiation of secondary acoustic waves and due to resonance damping.

In the case of radiative damping there are two possibilities: the amplitude of oscillation of a flux tube will grow if the energy of these oscillations and the energy of radiated waves have opposite signs, that is if tube oscillation has a positive energy, and the radiated sound wave has negative energy and vice versa. For example, the conditions for bending oscillations with positive energy to radiate a negative-energy sound wave have the form:

$$
a>s_{e} / \sqrt{\eta}, \quad u>s_{e}+\sqrt{a^{2}-s_{e}^{2} / \eta}
$$

and the corresponding growth rate is as follows (Ryutova, 1988):

$$
\frac{v_{\mathrm{rad}}^{\mathrm{b}}}{\omega}=\pi \mathrm{k}^{2} \mathrm{R}^{2} \frac{\left(c_{\mathrm{b}}-u\right)^{2}\left[\left(c_{b}-u\right)^{2}-s_{\mathrm{e}}^{2}\right]}{2 \mathrm{~s}_{\mathrm{e}}^{2} c_{\mathrm{b}}\left[(1+\eta) c_{b}-u\right]} .
$$


In the case of sausage oscillations the instability occurs under the following condition:

$$
\mathrm{u}>\mathrm{C}_{\mathrm{T}}+\mathrm{S}_{\mathrm{e}} \text {. }
$$

The corresponding growth rate has the form:

$$
\frac{v_{\mathrm{rd}}^{T}}{\omega}=\frac{\pi \mathrm{k}^{2} \mathrm{R}^{2}}{4 \eta} \frac{\mathrm{c}_{\mathrm{T}}^{2}\left(\mathrm{c}_{\mathrm{T}}-\mathrm{u}\right)^{2}}{\mathrm{a}^{4}} .
$$

Recently, similar results were obtained by Hollweg et al., 1989, who studied the effects of velocity shear on resonance absorption of incompressible MHD surface waves and indicated the importance of this effect for the development of turbulence in regions of strong velocity shear.

\section{Explosive Instability of Negative Energy Waves}

A specific nonlinear instability, an explosive instability, occurs in a system which contains waves with energies of different signs. A distinctive feature of an explosive instability is that the amplitudes of the interacting waves reach infinitely large values in a finite time. As an example, we present results for three wave interaction under the assumption that only one wave, say the sausage one is excited in the system at the origin, and the time scale and amplitudes of the two other waves (kink modes with $m=-1$ and $m=1$ ) are determined by the thermal noise. The conditions for explosive instability for this three-wave process are the following matching conditions:

$$
\omega_{\mathrm{T}}+\omega_{\mathrm{b}_{+}}+\omega_{\mathrm{b}_{-}}=0, \quad \mathrm{kT}_{\mathrm{T}}+\mathrm{k}_{\mathrm{b}_{+}}+\mathrm{k}_{\mathrm{b}_{-}}=0, \quad\left|\mathrm{~m}_{\mathrm{T}} \mathrm{I} \pm\right| \mathrm{m}_{\mathrm{b}_{+}}| \pm| \mathrm{m}_{\mathrm{b}_{-}} \mid=0 .
$$

The - and + with the subscript b correspond to waves which are travelling downstream and upstream. The third condition in set (11) corresponding to the conservation of the angular momentum of the waves can be easily satisfied since for the sausage mode $\mathrm{m}_{\mathrm{T}}=0$. The kink mode can be either linearly polarized, that is it can be a mixture of waves with $m=+1$ and $m=-1$, or circularly polarized in opposite directions. To satisfy the first condition in set (11) which corresponds to energy conservation it is obvious that at least one of the waves has to be the negative energy wave. As was mentioned above, the sausage mode is always a positive energy wave. Of the two kink modes the wave travelling downstream has a negative energy and negative wave number. Note, that in analyzing nonlinear processes the sign of the frequency is assumed to correspond to the sign of the energy, so $\omega_{\mathrm{b}}<0$. When this approach is taken, it is simple to verify that the matching conditions (11) are compatible if the following inequality holds:

$$
u \geq u_{\text {expl }}=\frac{c_{T}}{1+\eta}+\left[a^{2} \eta-c_{T}^{2} \frac{\eta\left(\eta^{2}+3 \eta+3\right)}{1+\eta^{2}}\right]^{1 / 2} .
$$

The value of $u_{\text {expl }}$ lies below the limit of hydrodynamic instability and just gets into the interval (10) corresponding to the existence of negative energy waves. Thus, under the condition

$$
a \sqrt{\eta}<u<u_{\text {expl }}
$$

the three wave interaction (note that two of them might be at the level of noise) can be explosive; in the initial stage of evolution of the explosive instability amplitudes of interacting waves grow exponentially, and the typical growth rate is $k_{T} v_{T} \sim$ in order of magnitude, where $v_{T} \sim$ is the velocity amplitude of the tube's boundary for sausage 
oscillations. After a time of the order of several times the reciprocal of the growth rate, at which the amplitudes of all three waves have become equal in order of magnitude, the amplitudes begin a power-law growth in accordance with

$$
v_{\sim} \sim 1 /\left(t-t_{0}\right),
$$

where to is the «explosion» time, which is also of the order of $\left(k_{T} v_{T}\right)^{-1}$. So, formally, in a time to the amplitudes of all three waves achieve infinitely large values. At this strongly nonlinear stage higher-order nonlinear processes are, of course, involved. These processes can limit the growth of amplitudes at a finite level. The further development of explosive instability depends on the character of these nonlinear processes as well as the dispersive properties of the system (see, for example, Weiland and Wilhelmsson, 1977). The earliest noninear effect which turns on is the nonlinear phase shift which leads to a breaking of the synchrony in time. Note, that the inhomogeneities of the medium lead to a breaking of «synchrony» in space. The stabilization of the explosive instability by the nonlinear phase shift can result in the formation of a solitary wave. Very important is the influence of the second dispersive effects, which lead to rapid oscillations (ripplings) superimposed on the main nonlinear solution. The interaction between new oscillations can again give rise to explosive growth of their amplitudes. This can happen of the nonlinear frequency shift counteracts the stabilization by second-order dispersive effects. Thus, in this case an «explosion» or fast and efficient energy release takes place.

In the opposite case, where the nonlinear phase shift enhances the stabilization due to dispersive effects there appear new solitons. There are situations (for example, due to a weak imbalance between the two stabilizing effects) when solitons with explosively growing amplitudes appear.

A soliton solution in flux tubes (in the absence of shear flows) was found earlier by Roberts and Mangeney (1982) by the traditional way of using the dispersion relation of tube oscillations keeping higher-order terms with respect to $(\mathrm{kR})^{2}$. Today the problem of solitons in flux tubes is a current interest of many explorers.

\section{5. «Magnetosonic Flows»}

A specific nonlinear effect which can play an essential role in the dynamics of magnetic structures and in their evolution in time consists of the generation of secondary plasma flows by oscillating flux tubes (Ryutova, 1986). This effect is similar to the effect of «acoustic flows» or «quartz wind» (Eckart's flows) in usual gasdynamics (see, for example, Nyborg, 1965), but its picture in magnetohydrodynamics appears to be much richer. These effects are caused by the presence of the boundary between the plasma and magnetic field due to the appearance of additional tangential tensions at the boundary. There are mainly two reasons leading to the generation of «magnetosonic flows». First is the action of the ponderomotive force on a plasma. This mechanism is not connected with the absorption of oscillation or with any dissipative effect. The second mechanism is just connected with the absorption of momentum and angular momentum of oscillations propagating along the flux tube. The absorption of longitudinal momentum (nonzero angular momentum can be transferred by circularly polarized bending oscillations) causes stationary convection across the flux tube (rotational mass flows inside and outside the tube). Moreover, the action of this mechanism leads also to current drive. The geometry of currents corresponds to the geometry of secondary flows.

The equation for the stationary velocity field of generated secondary flows $\vec{u}$ has the form:

$$
\rho v \nabla^{2} \overrightarrow{\mathrm{u}}=-\nabla\left(\mathrm{p}+\frac{\mathrm{B}^{2}}{8 \pi}\right)+\overrightarrow{\mathrm{f}},
$$

where $v$ is the kinematic viscosity and $\overrightarrow{\mathrm{f}}$ is the ponderomotive force acting on unit volume: 


$$
\vec{f}=-\left\langle\rho^{\prime} \frac{d \vec{v}^{\prime}}{d t}\right\rangle+\frac{1}{c}\left\langle\left[\vec{j}^{\mathbf{B}^{\prime}}\right]\right\rangle
$$

Here a prime denotes perturbed quantities in linear oscillations and is defined from the linearized MHD-equations corresponding to one of the modes (kink or sausage).

One can see from Eq. (13) that convective motions can arise only if

$$
\operatorname{rot} \overrightarrow{\mathbf{f}} \neq 0
$$

Otherwise, the ponderomotive force leads only to an insignificant redistribution of plasma parameters inside the flux tube. The magnitude of $f$ becomes especially large in the Alfven resonance layer where quite strong convective motions can arise.

The most essential consequence of the effect of secondary flows is that it affects the evolution of flux tubes and actually determines their lifetime. There are two different regimes of the evolution of a flux tube. During the action of the ponderomotive force a levelling of density and magnetic field along the field lines of this force takes place. The field of forces as well as the character of secondary flows and hence the further evolution of the flux tube depend on the relation between the duration $\mathrm{T}$ of the primary wavetrain and the time of establishing a viscous flow $\tau_{v} \sim R^{2} / v$.

In the case of a long coherent wavetrain when $T>\tau_{v}$ the equalization of density and magnetic fields leads to the «splitting» of the flux tube into four independent flux tubes. Gravity affects in this process (in the regions of small pressure scale height) can cause the separation of new flux tubes from each other and thus the formation of a «magnetic tree».

In the opposite case of a sequence of mutually incoherent short wavetrains with $\mathrm{T}<\tau_{\mathrm{v}}$, a kind of stochastic motion is induced which results in a diffusive broadening of the tube and ends up in a complete «dissolving» of the flux tube in the ambient plasma.

As was mentioned above the second mechanism of the generation of plasma flows and current drive is connected with the absorption of oscillations. Formally, the expression of the force has the same form as Eq. (14), but now the terms directly connected with the absorption are taken into account. Note, that besides the absorption due to the usual dissipative effects nondissipative mechanisms peculiar to flux tubes (and described above) are very efficient. For example, in the case of resonance damping when the energy of bending oscillations is pumping into the resonance layer where the dissipation occurs, the whole momentum of oscillations is transferred to the plasma in a narrow layer, causing a strong upward mass flow along the magnetic field. The same holds in the case of absorption of angular momentum when strong convection across the flux tube takes place. The corresponding force can be estimated as follows:

$$
f_{z} \sim v_{\text {res }} \frac{B^{2}}{4 \pi} \frac{\left|v^{\prime}\right|^{2}}{a^{2} R} .
$$

As was mentioned above, unlike the usual gasdynamics, in magnetic flux tubes the generation of plasma flows is accompanied by current drive. The quantitative solution of the problem is based on the two-fluid approach, where the current density is

$$
\vec{j}=\operatorname{en}\left(\overrightarrow{v_{i}}-\overrightarrow{v_{e}}\right) \text {. }
$$

Here $v_{i}$ and $v_{e}$ are the ion and electron velocities and $n$ is the plasma concentration. Twofluid MHD leads to the following equation for the current density:

$$
\frac{\partial \vec{j}}{\partial t}=e \frac{m_{e} \vec{f}_{i}-m_{i} \vec{f}_{e}}{m_{i} m_{e}}-\frac{e^{2} n}{\sigma} \frac{m_{i}+m_{e}}{m_{i} m_{e}} \vec{j}
$$


Here all the definitions are standard and $\vec{f}_{i}$ and $\vec{f}_{e}$ are the forces acting on ions and electrons, respectively. Note that the main contribtuion to the effect of current drive (as is clear from Eq. (15)) comes from the force $\overrightarrow{f_{e}}$ acting on the electrons. In the stationary case we have simply

$$
\vec{j}=\sigma\left(\vec{E}-\overrightarrow{f_{e}} / e\right)
$$

The detailed calculation of the force $\vec{f}_{\mathrm{e}}$ needs a kinetic approach and is too complicated, but in the problem under consideration there are circumstances which make the problem easy. The point is that in the longwave limit the particle mean free path (in a highly collisional solar plasma) 1 is much less than the wavelength of flux tube oscillations $\lambda: 1 \ll \lambda \sqrt{\mathrm{m}_{d} / \mathrm{m}_{\mathrm{i}}}$. This means that the perturbations of ion and electron temperatures as well as perturbations of ion and electron pressures in fluxtube oscillations are equal:

$$
\mathrm{p}_{\mathrm{i}}=\mathrm{pe} \text {. }
$$

So, from the momentum conservation law it is clear that half of the wave momentum is transferred to electrons and half to ions (Ryutov and Ryutova, 1989).

Therefore, for the mean force acting on the electron gas we can write:

$$
\overrightarrow{\mathrm{f}_{\mathrm{e}}}=\frac{1}{2} \overrightarrow{\dot{p}} \text {. }
$$

Here $\overrightarrow{\boldsymbol{P}}$ is the longitudinal momentum of tube oscillations:

$$
\dot{\mathcal{P}}=\frac{\gamma}{\mathrm{v}_{\varphi}} \mathrm{W},
$$

where $\gamma, v_{\varphi}$ and $W$ are the damping rate, phase velocity and energy density of the oscillations which causes the corresponding current drive. For example, an estimate of the current which can be generated due to the absorption of bending oscillations in the resonance layer is

$$
\mathrm{j}_{\mathrm{z}} \sim \mathrm{v}_{\mathrm{res}} \frac{\mathrm{eW}}{2 \mathrm{vm} \mathrm{m}_{\mathrm{e}}} \frac{\mathrm{R}}{\mathrm{l}} .
$$

We used here the model of a flux tube with a diffuse boundary of width $l ; v_{\varphi}=c_{b}$ is determined by Eq. (1) and $\gamma=v_{\text {res }}$ by Eq. (5).

The same considerations hold for the oscillations with circular polarization. In this case besides momentum the wave carries angular momentum. Now, the absorption of angular momentum leads to the generation of azimuthal currents.

\section{Summary}

In the present paper an attempt is made to describe some basic plasma effects which can be responsible for particular active processes in the Sun. There is a scheme of these effects and the links between them. Many links in this picture have yet not been found. And, of course, the picture itself is far from complete. But the most important next step is to find the quantitative relevance of these effects to particular observational data. 


\section{References}

Beckers, J. M. and Schröter, E. H., 1968, Solar Phys., 4, 142.

Chen, L. and Hasegawa, A., 1974, J. Geophys. Res., 79, 1024.

Coppi, B., Rosenbluth, M. N. and Sudan, R., 1969, Ann. Phys., 55, 207.

Defouw, R. J., 1976, Ap. J., 209, 266.

Dikasov, V. M., Rudakov, L. I. and Ryutov, D. D., 1965, JETR, 48, 913.

Frazier, F. N., 1971, in Proc. IAU Symposium No. 43, 93, R. Howard , ed.

Grigorjev, V. M. and Kuklin, G. V., 1971, Ibid.

Gopasyuk, S. I. and Tsap, T. T., 1971, Ibid.

Heyvaerts, J. and Priest, E., 1983, Astr. Ap., 117, 220.

Hollweg, J., 1979, Solar Phys. 62, 227.

Hollweg, J., Yang, G., Cadez, V. M. and Gakovic, 1989, Ap. J.

Howard, R., 1959, Ap. J., 130, 193.

Howard, R. and Stenflo, J., 1972, Solar Phys., 22, 402.

Ionson, J. A., 1978, Ap. J. 226, 650.

Kadomtsev, B. B., Mikhailovsky, A. B. and Timofeev, A. V., 1964, JETP, 47, 2267.

Livingston, W. and Harvey, J., 1969, Solar Phys., 10, 294.

Nyborg, W. L., 1965, in Physical Acoustics, Vol. 2, Part B, Properties of Polimers and Nonlinear Acoustics, Academic Press.

Priest, E. R., 1982, Solar Magnetohydrodynamics, Dodrecht, D. Reidel Publ. Co.

\section{Discussion}

UBEROI: What are the time scales involved in the nonlinear effects? How do these compare with the resonant and radiative damping time scales?

RYUTOVA: Several inverse damping rates, but to be more strict we have to specify which of the nonlinear effects we are dealing with. For example, in a case of secondary flows the duration of the incident wave train and the time of establishing viscous flows are involved as well.

PRIEST: (i) Do you think nonlinear effects are more important in the photosphere than the corona, where the Alfven Mach number is larger? (ii) Because the observed turbulent or wave flows are so small compared with the Alfven speed, do you think that coronal heating by waves is essentially a linear process? (iii) What effects do you expect to saturate your explosive instability in practice?

RYUTOVA: (i) No, and I mean no for all the different effects which I've considered today. Almost for all of them there is not only the question of the Alfven Mach number. For generation of secondary plasma flows and current drive the most important parameters are the duration of the incident wave train and the viscosity of the ambient plasma. The phenomena of splitting of magnetic structures (I do not mean the photospheric flux tubes only) and their diffusive vanishing must be common phenomena for different regions where the appropriate conditions are fulfilled. There is, of course, the coalescence effect, but it is out of the frame of this talk. For negative energy waves the region of their existence can be characterized by plasma $\beta$ as follows: $\sqrt{\frac{\beta}{1+\beta}} a<u<\sqrt{\frac{1+2 \beta}{1+\beta}}$ a, which shows that for low $\beta$ the lower threshold for critical shear velocity can be very low which makes a low $\beta$ region very favourable for negative energy waves and for all accompanied phenomena. With increasing $\beta$ the critical velocity threshold increases and the region of existence of negative energy waves becomes more narrow. (ii) I do not think so. The general philosophy and practice show that nonlinear effects are invaluable for efficient heating, at least when waves are involved. (iii) As I already mentioned, the nonlinear phase shift which leads to the breaking of the synchrony in time is the earliest effect which can saturate the explosive instability. 
ROBERTS: You have mentioned several mechanisms for the generation of waves in tubes. Which mechanism do you think is likely to be the most important in photospheric flux tubes, and do you expect impulsive phenomena to occur?

RYUTOVA: The most important effect for the excitation of photospheric flux tube oscillations is the resonance absorption of sound waves by flux tubes which takes place if the Cerenkov condition is fulfilled: $s=v_{\varphi} \cos \theta$ ( $s$ is sound speed, $v_{\varphi}$ is the phase velocity of tube waves and $\theta$ is an angle between the sound propagation and the tube axis). Besides that, a very important effect is noncollinearity of flux tubes (Ryutov and Ryutova, 1976). For excitation of negative energy waves the presence of shear flows along flux tubes is necessary. Concerning impulsive phenomena, as you have shown they can occur and I agree with that. 